\title{
A 7-LOCAL IDENTIFICATION OF THE MONSTER
}

\author{
C. W. PARKER AND C. B. WIEDORN
}

\begin{abstract}
We identify the monster from two of its 7-constrained maximal 7-local subgroups.
\end{abstract}

\section{§1. Introduction}

All groups considered in this article are finite. Suppose that $X$ is a group and $p$ is a prime. Then $X$ is $p$-constrained if $C_{X}\left(O_{p}(X)\right) \leq O_{p}(X)$. For a group $G, S \in S y l_{p}(G)$, and $T$ a non-trivial subgroup of $S, N_{G}(T)$ is called a $p$-local subgroup. We say that $G$ has local characteristic $p$ if every $p$-local subgroup is $p$-constrained and we say that $G$ has parabolic characteristic $p$ provided every $p$-local subgroup that contains $S$ is $p$-constrained. A $\mathcal{K}$-group is a group which has all its composition factors from among the known simple groups and a group $G$ is $\mathcal{K}$-proper if every proper subgroup of $G$ is a $\mathcal{K}$-group.

It is expected that the programme to identify the $\mathcal{K}$-proper groups of local characteristic $p$ orchestrated by Meierfrankenfeld, Stellmacher and Stroth (see [20]) will soon have a list of possible amalgams within such groups. Some of these amalgams will uniquely determine the target group via its $p$-local geometry (for example via the building if $G$ is expected to be a Lie type group in characteristic $p$ of rank at least 3 ). For other groups, where for example there are only two $p$-local subgroups containing a Sylow $p$-subgroup, other methods will be needed. In this paper we investigate one of these exceptional configurations. Our main theorem is as follows.

TheOREM 1.1. Suppose that $G$ is a $\mathcal{K}$-proper group, $S \in \operatorname{Syl}_{7}(G)$, $Z_{\beta}=Z(S)$ has order $7, Z_{\alpha}=Z_{2}(S)$ (the second centre of $S$ ) has order 49 and

(a) $N_{\beta}=N_{G}\left(Z_{\beta}\right) \sim 7_{+}^{1+4} .2 \cdot A l t(7) .6$ is 7-constrained; and

Received April 11, 2003.

Revised September 26, 2003.

2000 Mathematics Subject Classification: 20D05. 
(b) $N_{\alpha}=N_{G}\left(Z_{\alpha}\right) \sim 7^{2+1+2} \cdot G L_{2}(7)$ is 7 -constrained.

Assume that $O_{7}\left(\left\langle N_{\alpha}, N_{\beta}\right\rangle\right)=1$. Then $G$ is isomorphic to the monster, the largest sporadic simple group. In particular, $G$ is of parabolic characteristic 7 but not of local characteristic 7 .

In Theorem 1.1 we have used the Atlas [5, page $\mathrm{xx}$ ] notation for group extensions. We also use the Atlas [5] notation for the sporadic simple groups except that the baby monster is denoted by $B M$ rather than $B$. Cyclic groups of order $n$ will be denoted by $n$ or $\mathbb{Z}_{n}$. To avoid confusion with Lie type groups of type $A$ and $D$, we use $A l t(n)$ and $\operatorname{Sym}(n)$ for the alternating and symmetric groups of degree $n$, respectively, and $\operatorname{Dih}(n)$ for the dihedral group of order $n$. We use $p_{+}^{1+2 n}$ to denote the extraspecial group of order $p^{1+2 n}$ and exponent $p$ when $p$ is odd and, when $p=2$, the extraspecial group of order $2^{1+2 n}$ which has elementary abelian subgroups of order $2^{1+n}$. We write $G \sim A . B \ldots Z$ or say that $G$ has shape $A . B \ldots Z$ when $G$ has a normal series with factors of shape $A, B, \ldots, Z$. Thus, for example, $G \sim 7_{+}^{1+4} .2 \cdot A l t(7)$ indicates that $G$ contains proper normal subgroups of order $7,7^{5}, 2.7^{5}$ and $2.7^{5} .|A l t(7)|$. It also indicates that the normal subgroup $Q$ of order $7^{5}$ is an extraspecial group of exponent 7 and that $G / Q$ is isomorphic to a non-split extension of $A l t(7)$ by a cyclic group of order 2 . The notation $G \sim 7^{2+1+2} . G L_{2}(7)$ indicates that $G$ contains proper normal subgroups of orders $7^{2}, 7^{3}$, and $7^{5}$ but nothing further about the action of $G / O_{7}(G) \cong G L_{2}(7)$ on $O_{7}(G)$ is meant to be implicit in the notation. A p-element $x \in G$ is called $p$-central if $C_{G}(x)$ contains a Sylow $p$-subgroup of $G$.

The amalgam $\mathcal{A}$ consisting of the triple $\left(N_{\alpha}, N_{\beta}, N_{\alpha} \cap N_{\beta}\right)$ and the two inclusion mappings is an example of a symplectic amalgam and appears as $\mathcal{A}_{48}$ in [22, Table 1.8]. Notice that we have not assumed that $\mathcal{A}$ is isomorphic to the corresponding amalgam in $M$ (though it may well be) nor have we assumed that $G$ is generated by $N_{\alpha}$ and $N_{\beta}$.

Our strategy for identifying the monster from this 7-local information is to determine the structures of the centralizers of the involutions in $G$. Thus our objective is to find 2 involutions, one of which has 2-constrained centralizer of shape $2_{+}^{1+24} \cdot C_{1}$ and the other one has centralizer $2 \cdot B M$. Once this has been done, and after noting that the monster sporadic simple group possesses two 7-local subgroups as described in Theorem 1.1 (see [25] or [5, page 234]), the main result follows from [12]. As part of our proof 
we present some elementary results about $\mathcal{K}$-groups which characterize certain of the simple $\mathcal{K}$-groups by the structure of the centralizer of elements of order 7 or by the structure of their Sylow $p$-subgroups. These results are included in Section 2. Finally we mention that the monster has been characterized from the amalgam of its 2-local subgroups by Ivanov [15] and from the amalgam of its 3-local subgroups by Ivanov and Meierfrankenfeld [16]. These two results actually show that the universal completion of the amalgam under investigation is isomorphic to the monster.

There are other exotic amalgams related to the large sporadic simple groups. Four examples occur with $p=5$ and are listed as $\mathcal{A}_{20}, \mathcal{A}_{21}, \mathcal{A}_{46}$ and $\mathcal{A}_{53}$ in [22, Table 1.8]. These four amalgams are related to $H N, B M, L y$, and $M$, respectively, and are the subject of [21], [23], and [24]. A further remarkable example which occurs in the monster has $N_{\beta} \sim 13_{+}^{1+2} .12 . S y m(4)$ and $N_{\alpha} \sim 13^{2} .4 . L_{2}(13) .2$. With respect to this amalgam the monster is of parabolic characteristic 13; unfortunately, as yet we have no idea how to characterize $M$ from these two subgroups. The strategies used in [21], the present paper, [23] and [24] will not work in this particular case since the largest elementary abelian 13-subgroup in the centralizer of an involution in $N_{\alpha}$ and $N_{\beta}$ has order 13. Thus the critical method of proof used in Propositions 5.6 and 6.5 to control $O_{p^{\prime}}\left(C_{G}(t)\right)$ for an involution $t$ fails.

Dr. Corinna Wiedorn died on 25th February 2005 in Lingen, Germany. Her contribution to our mathematical community is greatly missed.

\section{§. Preliminaries}

This section primarily contains characterizations of $\mathcal{K}$-groups by their 7-local structure. However, we begin with a result concerning the 4-dimensional $G F(7)$-module for $G=2 \cdot A l t(7) .2$ which, as we shall see, appears in $N_{\beta}$ as $O_{7}\left(N_{\beta}\right) / Z_{\beta}$.

Firstly we note that the smallest $G F(7)$ representation of $A l t(7)$ is 6 dimensional (see [17, page 15]). So $G$ contains a subgroup isomorphic to the non-split extension $2 \cdot \operatorname{Alt}(7)$. Furthermore, as the multiplicative group of $G F(7)$ has no elements of order 4 , we have $G / Z(G) \cong \operatorname{Sym}(7)$. We note also before we start that the smallest dimension of a faithful $2 \cdot A l t(7) .2$ module over $G F(7)$ is 4 . Again this well-known fact can be read, for example, from [17, page 15$]$. 
Lemma 2.1. Let $G \sim 2 \cdot$ Alt(7).2, $V$ be a faithful irreducible 4-dimensional $G F(7) G$-module, and denote by $\mathcal{P}(V)=\{W \leq V \mid \operatorname{dim} W=1\}$ the set of one-dimensional subspaces of $V$.

(1) Let $x \in G, o(x)=3$. If $x$ projects to a 3 -cycle in $G / Z(G) \cong \operatorname{Sym}(7)$, then $C_{V}(x)=0$. If $x$ projects to a product of two disjoint 3 -cycles in $G / Z(G)$, then $\operatorname{dim} C_{V}(x)=2$.

(2) If $H \leq G$ with $H \cong S L_{2}(7)$, then $H$ acts irreducibly on $V$.

(3) $G^{\prime} \cong 2 \cdot A l t(7)$ has just two orbits on $\mathcal{P}(V)$. They have lengths 120 and 280 and stabilizers $2 \times 7: 3$ and $2 \times 3 \times 3$, respectively.

(4) $G^{\prime}$ has just two orbits on $V^{\#}$. They have lengths 720 and 1680 and stabilizers of order 7 and 3 , respectively.

(5) The orbits in (3) and (4) are preserved by $G$, the corresponding stabilizers being $2 \times 7: 6$ and $2 \times 3 \times \operatorname{Sym}(3)$ in (3) and $7: 2$ and $\operatorname{Sym}(3)$ in (4).

(6) Let $P=\langle x, y\rangle \in S_{y l}(G)$ such that $V=C_{V}(x) \oplus C_{V}(y)$. Let $W \in$ $\mathcal{P}(V), W \leq C_{V}(x)$. Then $N_{G}(W)$ acts irreducibly on $C_{V}(y)$. In particular, if $W \leq W_{1} \leq V$ such that $W_{1}$ is $N_{G}(W)$-invariant and $\operatorname{dim} W_{1}=3$ then $W_{1}=W+C_{V}(y)$.

(7) For $S \in S y l_{7}(G), \operatorname{dim} C_{V}(S)=\operatorname{dim} V /[V, S]=\operatorname{dim}[V, S] /[V, S, S]=$ 1 .

Proof. First of all as $V$ is a faithful module for $G$, we note that $\langle z\rangle=$ $Z=Z(G)$ inverts each vector of $V$. This fact will be used without further comment below.

(1) Let $x$ and $y$ be elements of order 3 projecting to (567) and (123) in $G / Z(G)$, respectively, and suppose $W=C_{V}(x) \neq 0$. Let $Q$ be the preimage in $G$ of the subgroup $\langle(12)(34),(13)(24)\rangle \leq A l t(7)$. Then $Q \cong Q_{8}$ and $\langle Q, y\rangle \cong Q_{8}: 3$. Since $[\langle Q, y\rangle, x]=1,\langle Q, y\rangle$ normalizes $W$, and since $Z \leq Q$ we see that $\langle Q, y\rangle$ acts faithfully and irreducibly on $W$ and on $V / W$ and that $\operatorname{dim} W=\operatorname{dim} V / W=2$. Since $\langle Q, y\rangle=\left\langle y^{Q}\right\rangle$, we have that $\operatorname{dim} C_{W}(y)=\operatorname{dim} C_{V / W}(y)=1$. Set $X=\langle x, y\rangle$. Then $X \in \operatorname{Syl}_{3}(G)$, $\operatorname{dim} C_{V}(X)=1$, and consequently

$$
V / C_{V}(X)=\bigoplus_{u \in X^{\#}} C_{V / C_{V}(X)}(u)
$$

has dimension 3. Since $C_{V}(X)$ is normalized by $N_{G}(X)$, since $x$ and $y$ as well as $x y$ and $x y^{-1}$ are conjugate in $N_{G}(X)$, and since $\operatorname{dim}_{V / C_{V}(X)}(x)=$ 
1, we infer that $\operatorname{dim} C_{V / C_{V}(X)}(u) \geq 1$ for all $u \in X^{\#}$. This means that $\operatorname{dim} V / C_{V}(X)>3$, a contradiction. Thus we have that $C_{V}(x)=0$ and as a consequence we have $\operatorname{dim} C_{V}(x y)=2$. Hence (1) holds.

(2) Suppose that (2) is false and let $W \leq V$ be such that $W$ is normalized by $H$. Since $Z \leq H$, we have $\operatorname{dim} W=2$ and $H$ acts faithfully in its natural representation on $W$ and $V / W$. Since 3-elements of $S L_{2}(7)$ act fixed point freely on the natural $S L_{2}(7)$-module, they act fixed point freely on $V$. But the only 3-elements of $G$ which are conjugate into $H$ are those which correspond to a product of two disjoint 3-cycles in $A l t(7)$ and these elements have fixed points by (1). Therefore, (2) holds.

(3) and (4) Let $P \in \operatorname{Syl}_{3}\left(G^{\prime}\right)$. Then by (1) there are $x \in P^{\#}$ and $W \in \mathcal{P}(V)$ such that $[W, x]=0 \neq[W, P]$ and such that $W$ is normalized by $P$. Thus, $\langle z, P\rangle \leq N_{G^{\prime}}(W),\langle z, P\rangle \cong 2 \times 3 \times 3$, and $\langle z, P\rangle \cap C_{G^{\prime}}(W)=\langle x\rangle$. In particular, $\langle z, P\rangle$ realizes the full automorphism group $\mathbb{Z}_{6}$ on $W$. Hence, if $N_{G^{\prime}}(W)>\langle z, P\rangle$ then $C_{G^{\prime}}(W)>\langle x\rangle$. Now any subgroup of $\operatorname{Alt}(7)$ properly containing a Sylow 3-subgroup also contains an involution. But all involutions in $\operatorname{Alt}(7)$ lift to elements of order 4 in $2 \cdot \operatorname{Alt}(7)$, whence they square to $z$ and so cannot be contained in $C_{G^{\prime}}(W)$. It follows that $W$ is a representative for an orbit of length 280 for $G^{\prime}$ on $\mathcal{P}(V)$ and that the structures of $N_{G^{\prime}}(W)$, and $C_{G^{\prime}}(W)$ are as claimed in (3) and (4).

Since $G^{\prime}$ has an orbit of length 280 on $\mathcal{P}(V)$, it follows that any other orbit of $G^{\prime}$ on $V$ has length at most $400-280=120$.

Let $S \in \operatorname{Syl}_{7}\left(G^{\prime}\right)$. Then there is $U \in \mathcal{P}(V)$ with $U \leq C_{V}(S)$. Certainly $U$ is in a different $G^{\prime}$ orbit than $W$. Suppose that two distinct Sylow 7-subgroups of $G^{\prime}$ centralize $U$. Then $U$ is centralized by a subgroup isomorphic to $S L_{2}(7)$ or by $G^{\prime}$. This contradicts (2) or the fact that $V$ is an irreducible $G^{\prime}$-module. Hence we infer that $U$ is centralized by a unique Sylow 7 -subgroup and so $N_{G^{\prime}}(U) \leq N_{G^{\prime}}(S) \cong 2 \times 7: 3$. Since $\left|G^{\prime}: N_{G^{\prime}}(S)\right|=120$, we conclude that $N_{G^{\prime}}(U)=N_{G^{\prime}}(S)$. Clearly, $z \in N_{G^{\prime}}(U) \backslash C_{G^{\prime}}(U)$ and by (1) and the previous paragraph $U$ is not centralized by a 3 -element, either. So (3) and (4) are proved.

(5) Since 400 does not divide $|\operatorname{Sym}(7)|$, we see that $G \sim 2 \cdot \operatorname{Sym}(7)$ must preserve the orbits of $G^{\prime}$. Since $\left|G: G^{\prime}\right|=2$ and, as we have seen that, for any $X \in \mathcal{P}(V), N_{G^{\prime}}(X)$ realizes $\operatorname{Aut}(X)$ on $X$, it is easy to deduce from $(3),(4)$, and the structure of a group of shape $2 \cdot \operatorname{Sym}(7)$ that the structures of $N_{G}(X)$ and $C_{G}(X)$ are as stated.

(6) In the proofs of (3), (4), and (5) we have seen that $N_{G}(W) \cong$ $2 \times 3 \times \operatorname{Sym}(3)$. Further, $N_{G}(W) \leq C_{G}(y)$ and so $N_{G}(W)$ acts on $C_{V}(y)$. Since $x$ acts fixed point freely on $C_{V}(y)$ and $C_{G}(W) \cong \operatorname{Sym}(3)$ we see that 
$C_{G}(W)$ cannot normalize a 1 -space in $C_{V}(y)$.

(7) Suppose that $S$ centralizes two subspaces $U, W \in \mathcal{P}(V)$. Then by (3) we have $W=U^{g}$ for some $g \in G^{\prime} \backslash N_{G^{\prime}}(S)$. So $S<\left\langle S, S^{g}\right\rangle \leq C_{G}(W)$ and this contradicts (3) and (4). Hence $\operatorname{dim} C_{V}(S)=1$. It follows from [1, Lemma 5.5, Theorem 6.4] that as an $S$-module $V$ embeds into the regular $S$-module of dimension 7. Since this module is uniserial the claims in (7) all hold.

Corollary 2.2. If $G \sim 2 \cdot$ Alt(7).2 acts faithfully on a 4-dimensional vector space over $G F(7)$, then $G \cong 2^{-} \operatorname{Sym}(7)$.

Proof. By Lemma 2.1(5) there is an involution $x \in G \backslash G^{\prime}$ such that $x$ centralizes a non-zero vector in $V$ and normalizes a Sylow 7-subgroup of $G$. The latter forces $x$ to map onto a product of three disjoint transpositions in $\operatorname{Sym}(7)$. In other words, the involutions of $\operatorname{Sym}(7)$ which lift to involutions in $G$ are the products of three disjoint transpositions whereas transpositions lift to elements of order 4 in $G$. By [5, page 236], this is just the definition of the group $2^{-} \operatorname{Sym}(7)$ (in contrast to $2^{+} \operatorname{Sym}(7)$ ).

The irreducibility of both conjugacy classes of subgroups isomorphic to $S L_{2}(7)$ in $2 \cdot \operatorname{Alt}(7)$ on $V$ proved in Lemma 2.1(2) will be a key point later and distinguishes the situation considered in this paper significantly from the corresponding 5-local case, which can be seen in the sporadic simple group discovered by Lyons. Indeed, in $L y$ we have the 5-constrained 5-local subgroup $5_{+}^{1+4} \cdot 2 \cdot A l t(6) .4$. So $2 . A l t(6)$ is acting on a 4 -dimensional $G F(5)$ space. Now $2 \cdot A l t(6)$ contains two conjugacy classes of subgroup $2 \cdot \operatorname{Alt}(5) \cong$ $S L_{2}(5)$. It turns out that the module restricted to one of these subgroups is irreducible and restricted to the other one it is an indecomposable extension of two natural modules for $S L_{2}(5)$ (see [21] for further details).

In the following theorem we determine all the simple $\mathcal{K}$-groups which have an extraspecial Sylow $p$-subgroup of order $p^{3}$ for some prime $p$. In this paper, of course, we will then just make use of the case $p=7$ but we will use the case $p=5$ in [24]. We remark that the simple groups with dihedral Sylow 2-subgroups have been determined by Gorenstein and Walter (see [10], [11]) independent from the $\mathcal{K}$-group hypothesis. A shorter proof has been established by Bender and Glauberman (see [2], [3]). It was shown by Brauer and Suzuki (see [4]) that there are no finite simple groups with quaternion Sylow 2-subgroups. It is also a pleasure at this stage to acknowledge the assistance of our referee who pointed out that our original 
proof of our next theorem could be dramatically shortened by citing $[9$, $4.10 .3(\mathrm{c})]$.

TheOREM 2.3. Let $G$ be a simple $\mathcal{K}$-group, $p$ a prime, and $S \in$ $\operatorname{Syl}_{p}(G)$. Assume that $S$ is extraspecial of order $p^{3}$. Then one of the following holds.

(1) $G \cong L_{3}(p)$ or $p$ is odd and $G \cong U_{3}(p)$.

(2) $p=2$ and $G \cong A l t(7)$ or $G \cong L_{2}(q)$ where $q \equiv \pm 7(\bmod 16)$.

(3) $p=3$ and $G \cong G_{2}(2)^{\prime} \cong U_{3}(3)$ or $G \cong G_{2}\left(r^{a}\right)$ for some prime $r$ where $r \equiv \pm 2, \pm 4(\bmod 9)$, a is not divisible by 3 , and $a>1$ if $r=2$.

(4) $p=3$ and $G \cong{ }^{2} F_{4}(2)^{\prime}$ or $G \cong{ }^{2} F_{4}\left(2^{a}\right)$, where $a \geq 3$ is odd and not divisible by 3 .

(5) $G$ is a sporadic group and

(i) $p=3$ and $G$ is one of $M_{12}, M_{24}, J_{2}, J_{4}, H e, R u$; or

(ii) $p=5$ and $G$ is one of $\mathrm{HS}, \mathrm{Co}_{3}, \mathrm{Co}_{2}, \mathrm{McL}, \mathrm{Ru}$, Th; or

(iii) $p=7$ and $G$ is one of $\mathrm{He}, \mathrm{O}^{\prime} N, \mathrm{Fi}_{24}^{\prime}$; or

(iv) $p=11$ and $G \cong J_{4}$; or

(v) $p=13$ and $G \cong M$.

Proof. For the case $p=2$ we simply refer to [4] and to [10, Theorem 2] (see also [6, page 462]). So from now on we assume $p$ is odd.

Suppose first that $G \cong A l t(m)$ for some $m$. Then $p>3$ since there are no alternating groups with Sylow 3 -subgroup of order $3^{3}$. Further, as $|S|=p^{3}$, we must have $3 p \leq m \leq 4 p-1$. But for such $m$ the Sylow $p$-subgroups of $A l t(m)$ are elementary abelian, generated by three disjoint $p$-cycles. So $G$ is not an alternating group.

If $G$ is a Lie type group in characteristic $p$ with associated root system $\Phi$, then, since the order of $S$ is at least $p^{a|\Phi| / 2}$, we deduce that $G$ is isomorphic to either $L_{3}(p)$ or $U_{3}(p)$.

Suppose now that $G$ is a group of Lie type defined over a field of order $r^{a}$ where $r$ is a prime with $r \neq p$. By a result due to Huppert [8, 15.21] the exponent of $S$ is $p$ and so $S$ contains $p+1$ maximal elementary abelian subgroups. Using $[9,4.10 .3$ (c)] we infer that $p=3$ and that $G$ is isomorphic to one of $A_{2}\left(r^{a}\right)$ with $r^{a} \equiv 1(\bmod 3),{ }^{2} A_{2}\left(r^{a}\right)$ with $r^{a} \equiv-1(\bmod 3)$, ${ }^{2} F_{4}\left(2^{a}\right)^{\prime}, G_{2}\left(r^{a}\right)^{\prime}$, or ${ }^{3} D_{4}\left(r^{a}\right)$. In the first two cases a Sylow 3-subgroup of the universal group of Lie type is contained in a subgroup $\left(r^{a}-1\right): \operatorname{Sym}(3)$ when $G \cong A_{2}\left(r^{a}\right)$ and in a subgroup $\left(r^{a}+1\right): \operatorname{Sym}(3)$ when $G \cong{ }^{2} A_{2}\left(r^{a}\right)$. 
Thus, a Sylow 3-subgroup of the simple group $G$ (which is the universal group factored by its centre of order 3 ) has order either $3^{2}$ or strictly greater than $3^{3}$. So these cases do not occur. Also, by [7, 10-1(4)] we have that $G \neq^{3} D_{4}\left(r^{a}\right)$. Therefore, to conclude the proof of (3) and (4) we only have to establish the assertions about $r$ and $a$. First let $G \cong{ }^{2} F_{4}\left(2^{a}\right)$. Then $a$ is odd since the groups ${ }^{2} F_{4}\left(2^{a}\right)$ are only defined for odd $a$. To prove that $a$ is not divisible by 3 , we examine each of the possibilities $a \equiv 0, \pm 1(\bmod 3)$ and look when

$$
2^{a} \equiv-1 \quad(\bmod 3), \quad \text { but } 2^{a} \not \equiv-1 \quad(\bmod 9) .
$$

If $a=3 b$ for some $b$ then $b$ must be odd (since $a$ is odd). So

$$
2^{a}=2^{3 b} \equiv 8^{b} \equiv(-1)^{b}=-1 \quad(\bmod 9) .
$$

Hence this case cannot occur. If $a=3 b+1$ then $b$ is even. So

$$
2^{a}=2^{3 b+1} \equiv 2 \cdot 8^{b} \equiv 2 \cdot(-1)^{b}=2 \quad(\bmod 9)
$$

and

$$
2^{a}=2^{3 b+1} \equiv 2 \cdot 2^{b} \equiv 2 \cdot(-1)^{b}=2 \equiv-1 \quad(\bmod 3) .
$$

Similarly, if $a=3 b+2$ then $b$ is odd. So

$$
2^{a}=2^{3 b+2} \equiv 4 \cdot 8^{b} \equiv 4 \cdot(-1)^{b}=-4 \quad(\bmod 9)
$$

and

$$
2^{a}=2^{3 b+2} \equiv 4 \cdot 2^{b} \equiv 1 \cdot(-1)^{b}=-1 \quad(\bmod 3) .
$$

So when 3 does not divide $a$, the Sylow 3 -subgroups of ${ }^{2} F_{4}\left(2^{a}\right)$ are at least of order $3^{3}$. As ${ }^{2} F_{4}\left(2^{a}\right) \geq{ }^{2} F_{4}(2)^{\prime} \geq L_{3}(3)$ (see [5, page 74]) they are seen to be extraspecial and (4) is shown.

The proof for $G_{2}\left(r^{a}\right)$ is an easy exercise and goes in the same way, distinguishing the cases $r \equiv \pm 1(\bmod 3)$ and $\operatorname{ord}_{3}\left(r^{a}\right)=1,2$. We leave the details for the reader and just mention that $G_{2}\left(r^{a}\right) \geq G_{2}(2)^{\prime} \cong U_{3}(3)$ for all $r$ (see [5, page 14] for $r=2$ and [18] for $r$ odd). This again shows that the Sylow 3-subgroups are extraspecial in the respective cases.

Finally, if $G$ is a sporadic group the statement follows by inspection of [9, pages 262-287]. This concludes the proof.

Corollary 2.4. Assume that $G$ is a simple $\mathcal{K}$-group and $S \in \operatorname{Syl}_{7}(G)$. If $N_{G}(S) \sim 7_{+}^{1+2}:(3: 6) \sim 7_{+}^{1+2}:(3 \times \operatorname{Sym}(3))$, then $G \cong H e$. 
Proof. Since $S$ is extraspecial of order $7^{3}, G \cong L_{3}(7), U_{3}(7), H e, O^{\prime} N$ or $F i_{24}^{\prime}$ by Theorem 2.3. The groups $L_{3}(7)$ and $U_{3}(7)$ have $N_{G}(S) / S \cong 6 \times 2$ and $N_{G}(S) / S \cong 48$, respectively. So $G$ is neither of these two groups. We now consult $[9$, Tables $5.3 \mathrm{~s}$ and $5.3 \mathrm{v}]$ to see that the only possibility is that $G \cong H e$.

TheOREM 2.5. Let $G$ be a simple $\mathcal{K}$-group and $S \in S_{\text {Syl }}(G)$. Assume that $|S|=7^{2}$ and, for any $x \in S^{\#}$, either

(1) $C_{G}(x) \cong 7 \times A l t(7)$, or

(2) $C_{G}(x) \cong 7 \times L_{3}(2)$ or $7 \times 2^{6} \cdot L_{3}(2)$,

and that $S$ contains elements of type (1) as well as of (one of the types in) (2). Then $G \cong C_{1}$ and the possibility that $C_{G}(x) \sim 7 \times 2^{6} . L_{3}(2)$ does not occur.

Proof. Again we examine each of the possibilities for $G$. If $G$ is an alternating group Alt(n), then, as $|S|=7^{2}, n \geq 14$. Taking $x$ to be a 7 -cycle we have $C_{G}(x) \cong A l t(n-7) \times 7$ and so $n=14$. Now take $x$ to be a permutation of cycle shape $7^{2}$. Then $C_{G}(x)=S$, a contradiction. Thus $G$ is not an alternating group.

Suppose that $G$ is a Lie type group defined in characteristic $r$. From $[9$, Theorem 4.9.6], for $x \in S$, the components of $C_{G}(x)$ are Lie type groups in characteristic $r$. Since $A l t(7)$ is not a Lie type group in any characteristic, we have a contradiction in this case.

Finally assume that $G$ is a sporadic simple group. Since $|S|=7^{2}$, by considering group orders we see that the only possibilities are $G \cong \mathrm{Co}_{1}$, $G \cong T h$ or $G \cong B M$. Consulting [9, Tables $5.3 \mathrm{l}, 5.3 \mathrm{x}$ and $5.3 \mathrm{y}$ ] and using the fact that $G$ has at least two conjugacy classes of cyclic subgroups of order 7 , we immediately obtain $G \cong C o_{1}$. Since $C o_{1}$ does not possess a 7-element with centralizer $7 \times 2^{6} \cdot L_{3}(2)$, the remaining part of the theorem follows.

Theorem 2.6. Assume that $G$ is a simple $\mathcal{K}$-group and $S \in \operatorname{Syl}_{7}(G)$. If $|S|=7^{2}$ and, for some $x \in S^{\#}, C_{G}(x) \cong 7 \times 2 \cdot L_{3}(4) .2$, then $G \cong B M$.

Proof. Once more we consider the possibilities for $G$. If $G \cong \operatorname{Alt}(n)$ with $n \geq 5$, then, as the minimal permutation representation of $2 \cdot L_{3}(4) .2$ has degree at least 21 , we have $|G|_{7} \geq 7^{3}$, a contradiction.

Suppose that $G$ is a Lie type group defined in characteristic $r$. Since $G$ is not of local characteristic $7, r \neq 7$. From [9, Theorem 4.2.2 (ii)], for 
$x \in S$, the components of $C_{G}(x)$ are Lie type groups in characteristic $r$. Since $2 \cdot L_{3}(4) .2$ is not a Lie type group in any characteristic, we have a contradiction in this case.

Finally assume that $G$ is a sporadic simple group. Then, as $|S|=7^{2}$, looking at $[9$, Tables $5.31,5.3 \mathrm{x}$ and $5.3 \mathrm{y}]$ we immediately see that $G \cong B M$.

LEMMA 2.7. Let $F$ be a group and $H$ be a non-trivial subgroup of $F$ such that $H \leq R$ whenever $R$ is a non-trivial subgroup of $F$ which is normalized by $H$. Then $F$ has a unique minimal normal subgroup $N, N$ is simple, and either

(1) F embeds into Aut $(N)$, or

(2) $H=N$ cong $\mathbb{Z}_{p}$ for some prime $p, C_{F}(N)$ is cyclic of order $p^{k}$ for some $k \geq 1$, and $F / C_{F}(N)$ embeds into $A u t\left(\mathbb{Z}_{p}\right)$, or

(3) $H=N \cong \mathbb{Z}_{2}$ and $F=C_{F}(N)$ is a quaternion group.

Proof. If $N$ and $M$ are minimal normal subgroups of $F$, then $1 \neq$ $H \leq N \cap M$ and so $N=M$. Therefore, $F$ has a unique minimal normal subgroup $N$.

Since $N$ is a minimal normal subgroup of $F, N$ is a direct product of simple groups, say, $N=N_{1} \times N_{2} \times \cdots \times N_{k}$. But as $H \leq N \leq N_{G}\left(N_{i}\right)$ for $1 \leq i \leq k$, we get $H \leq \bigcap_{i=1}^{k} N_{i}$ and so we conclude that $N$ is simple. If $N$ is a non-abelian simple group then (1) holds. So assume $H=N \cong \mathbb{Z}_{p}$ for some prime $p$. Set $K=C_{F}(N)$. Of course, $F / K$ embeds into $A u t\left(\mathbb{Z}_{p}\right)$. Let $S \in S y l_{r}(K)$ for some prime $r \neq p$. Then $H=N \leq C_{F}(S)$ and so $H \leq S$ by hypothesis, a contradiction. Hence $K$ is a $p$-group. Finally, if $x \in K$ is an element of order $p$ then again $H=N \leq C_{F}(\langle x\rangle)$ and so $H=N=\langle x\rangle$. This shows that $K$ contains a unique subgroup of order $p$. Now by [6, Theorem 4.10] either $N$ is cyclic and (2) holds or $p=2, F=K$ is a quaternion group, and (3) holds.

\section{§3. Some general properties of the amalgam}

In this section we set up some more notation and we prove some general properties of the amalgam $\mathcal{A}=\left(N_{\alpha}, N_{\beta}, N_{\alpha} \cap N_{\beta}\right)$ described in the hypothesis of Theorem 1.1. These results will be used without further reference in the remaining sections.

For $\gamma \in\{\alpha, \beta\}$ we set $L_{\gamma}=N_{\gamma}^{\infty}$ and $Q_{\gamma}=O_{7}\left(N_{\gamma}\right)$. Also, we usually write $N_{\alpha \beta}=N_{\alpha} \cap N_{\beta}$ and $L_{\alpha \beta}=L_{\alpha} \cap L_{\beta}$. The results in the next lemma 
follow from the information presented in the statement of Theorem 1.1 with a little help from Lemma 2.1.

LEMma 3.1. (1) $S=Q_{\alpha} Q_{\beta}, Z_{\beta} \leq Z_{\alpha} \leq Q_{\beta}$.

(2) $Q_{\beta}$ is extraspecial and $Q_{\beta} / Z_{\beta}$ is an irreducible module for $L_{\beta} / Q_{\beta} \cong$ $2 \cdot \operatorname{Alt}(7)$.

(3) $Q_{\alpha} \cap Q_{\beta}=C_{Q_{\beta}}\left(Z_{\alpha}\right)=\left[Q_{\alpha}, Q_{\beta}\right]$ and $Z_{\alpha} / Z_{\beta}=C_{Q_{\beta} / Z_{\beta}}(S)$.

(4) $Z_{\alpha}$ is elementary abelian, $\left|\Phi\left(Q_{\alpha}\right)\right|=7^{3}, \Phi\left(Q_{\alpha}\right) \leq Q_{\beta}$, and $Z_{\alpha}$ and $Q_{\alpha} / \Phi\left(Q_{\alpha}\right)$ are natural modules for $N_{\alpha} / Q_{\alpha} \cong G L_{2}(7)$; in particular, all elements in $Z_{\alpha}^{\#}$ are conjugate in $N_{\alpha}$.

(5) $N_{N_{\beta}}\left(Z_{\alpha}\right)=N_{\alpha \beta}=N_{N_{\alpha}}\left(Z_{\beta}\right)$ and $N_{\alpha \beta} / Q_{\alpha} \cong 7: 6 \times 6 \cong N_{\alpha \beta} / Q_{\beta}$.

(6) $L_{\beta}=C_{N_{\beta}}\left(Z_{\beta}\right)$; in particular, $N_{\beta}$ realizes the full automorphism group $\mathbb{Z}_{6}$ on $Z_{\beta}$.

(7) $N_{\beta}=L_{\beta} N_{N_{\alpha}}\left(Z_{\beta}\right)=L_{\beta} N_{\alpha \beta}$ and $N_{\beta} / Q_{\beta} \sim 2 \cdot(3 \times \operatorname{Sym}(7))$.

(8) $N_{\alpha}=L_{\alpha} N_{N_{\beta}}\left(Z_{\alpha}\right)=L_{\alpha} N_{\alpha \beta}$.

(9) $Q_{\beta}$ and $Q_{\alpha}$ are both characteristic subgroups of $S$.

(10) $Q_{\beta}$ contains representatives of at most two $G$-conjugacy classes of cyclic subgroups of order 7 .

Proof. (1) Since $O_{7}\left(\left\langle N_{\alpha}, N_{\beta}\right\rangle\right)=1$, the statements $S=Q_{\alpha} Q_{\beta}$ and $Z_{\beta} \leq Z_{\alpha}$ are immediate from the definitions. Furthermore, as $Z_{\alpha}=Z_{2}(S)$ and $Q_{\beta}$ is normal in $S, Z_{\alpha} \leq Q_{\beta}$.

(2) Since, by [17], the smallest dimension of a faithful representation of $2 \cdot \operatorname{Alt}(7)$ over $G F(7)$ is $4,(2)$ holds.

(3) Observing that $\left[Q_{\alpha}, Q_{\beta}\right] \leq Q_{\alpha} \cap Q_{\beta}$ and that the latter is of order $7^{4}$ the statement in (3) follows from (1), the fact that $N_{\beta}$ is 7 -constrained and Lemma 2.1(7).

(4) If $N_{\alpha} \leq N_{\beta}$, then $Q_{\beta} \leq O_{7}\left(\left\langle N_{\alpha}, N_{\beta}\right\rangle\right)=1$, which is a contradiction. Thus $N_{\alpha}$ does not normalize $Z_{\beta}$. In particular, $Z_{\alpha}$ is not cyclic, for otherwise $Z_{\beta}$ would be the unique cyclic subgroup of $Z_{\alpha}$ of order 7 and as such would be normal in $N_{\alpha}$. Furthermore, we see that $N_{\alpha} / Q_{\alpha} \cong G L_{2}(7)$ acts as $G L_{2}(7)$ on $Z_{\alpha}$. Since $\Phi\left(Q_{\alpha}\right) \geq\left[Q_{\beta} \cap Q_{\alpha}, Q_{\alpha}\right]=\left[Q_{\beta}, Q_{\alpha}, Q_{\alpha}\right]$ which has order $7^{3}$ by Lemma 2.1(7) and $Q_{\alpha} / \Phi\left(Q_{\alpha}\right)$ admits $N_{\alpha} / Q_{\alpha}$ faithfully, the remaining parts of (4) follow easily.

(5) The first claim follows directly from the definition of $N_{\alpha}$ and $N_{\beta}$ and (4) and the structure of $G L_{2}(7)$ imply the structure of $N_{\alpha \beta} / Q_{\alpha}$. The structure of $N_{\alpha \beta} / Q_{\beta}$ can be infered from the action of $G L_{2}(7)$ on the natural module $Q_{\alpha} / \Phi\left(Q_{\alpha}\right)$ involved in $Q_{\alpha}$ and (1). 
(6) A cyclic group of order 6 which normalizes $Z_{\beta}$ and acts faithfully on it can be observed in the quotient $N_{\alpha} / Q_{\alpha} \cong G L_{2}(7)$.

(7) The first statement follows from parts (5) and (6). Further, since $\operatorname{Aut}(\operatorname{Alt}(7)) \cong \operatorname{Sym}(7)$ either $N_{\beta} / Q_{\beta} \sim 2 \cdot(6 \times \operatorname{Alt}(7))$ or $N_{\beta} / Q_{\beta}$ is as claimed. But the first possibility obviously contradicts the structure of $N_{\alpha \beta} / Q_{\beta}$ stated in (5).

(8) To see this we note that $N_{\alpha}=L_{\alpha} N_{N_{\alpha}}(S)=L_{\alpha} N_{N_{\alpha}}\left(Z_{\beta}\right)$ by a Frattini argument.

(9) Because $|S|=7^{6}, Z_{\beta}=Z(S)$, and $Z_{\alpha} / Z_{\beta}=C_{Q_{\beta} / Z_{\beta}}(S)$, we have that $Q_{\beta}$ is the unique extraspecial subgroup of order $7^{5}$ in $S$. To see that $Q_{\alpha}$ is characteristic in $S$ we simply note that $Z_{\alpha}$ is characteristic in $S$ by its definition and that $Q_{\alpha}=C_{S}\left(Z_{\alpha}\right)$.

(10) By Lemma 2.1(3), $N_{\beta}$ has two orbits on the cyclic subgroups of $Q_{\beta} / Z_{\beta}$. Since for each subgroup $F \leq Q_{\beta}$ of order 49 containing $Z_{\beta}, Q_{\beta}$ has two orbits on the cyclic subgroups of $F$, we infer that there are $3 N_{\beta^{-}}$ conjugacy classes of cyclic subgroups in $Q_{\beta}$. One of these conjugacy classes consists of $Z_{\beta}$ and we have seen in (4) that $Z_{\beta}$ is $N_{\alpha}$-conjugate to a cyclic subgroup of $Q_{\beta}$ not contained in $Z_{\beta}$. Hence (10) is true.

We now exploit the previous lemma to deduce some information about the structures of $N_{\alpha}$ and $N_{\beta}$. We have that $N_{\alpha \beta} \sim S:(6 \times 6)$. Let $T$ be a fixed complement to $S$ in $N_{\alpha \beta}$. So $N_{\alpha \beta}=S T$ and $T \cong 6 \times 6$. Let $t_{1}, t_{2}, t_{3}$ be the three involutions in $T$. From the structure of $N_{\alpha}$ and $N_{\beta}$ we may assume that $t_{1} Q_{\beta} \in Z\left(N_{\beta} / Q_{\beta}\right)$ and $t_{2} Q_{\alpha} \in Z\left(N_{\alpha} / Q_{\alpha}\right)$. We set $J_{\beta}=C_{N_{\beta}}\left(t_{1}\right), K_{\beta}=J_{\beta}^{\infty}$ and, similarly, $J_{\alpha}=C_{N_{\alpha}}\left(t_{2}\right), K_{\alpha}=J_{\alpha}^{\infty}$. So

$$
\begin{aligned}
& J_{\beta} \sim(7 \times 2 \cdot \operatorname{Alt}(7)): 6, \quad K_{\beta} \cong 2 \cdot \operatorname{Alt}(7) \\
& J_{\alpha} \sim\left(7 \times S L_{2}(7)\right): 6 \quad \text { and } \quad K_{\alpha} \cong S L_{2}(7) \text {. }
\end{aligned}
$$

LEMmA 3.2. We have $t_{2}, t_{3} \in N_{\beta} \backslash L_{\beta}, t_{1}, t_{3} \in N_{\alpha} \backslash L_{\alpha}$. In particular, $\left\langle L_{\beta}, t_{2}\right\rangle=\left\langle L_{\beta}, t_{3}\right\rangle \cong 2^{-} \operatorname{Sym}(7)$.

Proof. Since the involutions in $A l t(7)$ lift to elements of order 4 in $2 \cdot \operatorname{Alt}(7), L_{\beta}$ does not contain an elementary abelian group of order 4 . Therefore the first statement holds for $N_{\beta}$. By Lemma 3.1(7) and Corollary 2.2 we have that $\left\langle L_{\beta}, t_{2}\right\rangle=\left\langle L_{\beta}, t_{3}\right\rangle \cong 2^{-} \operatorname{Sym}(7)$. Since $S L_{2}(7)$ contains a single involution, the lemma also holds for $N_{\alpha}$. 


\section{$\S 4$. The centralizer of a non 7-central element}

In this section we pick a certain subgroup of $S$ of order 7 and show that it is not contained in the center of any Sylow 7-subgroup of $G$. We prove that its centralizer in $G$ is isomorphic to $7 \times H e$ and so is not 7 -constrained. In particular, we note that $G$ is not of local characteristic 7 . The existence of the subgroup $7 \times H e$ in $G$ will be exploited in Sections 5 and 6 to obtain information about the 2-local structure of $G$.

Lemma 4.1. Let $U_{\alpha}=C_{Q_{\alpha}}\left(t_{2}\right)$. Then $\left|U_{\alpha}\right|=7, U_{\alpha} \leq Q_{\beta}$, and $U_{\alpha}$ is not 7-central in $G$.

Proof. As $Q_{\alpha}$ involves two natural modules for $N_{\alpha} / Q_{\alpha}$ and $t_{2}$ acts fixed point freely on such modules and $L_{\alpha}$ centralizes $\Phi\left(Q_{\alpha}\right) / Z_{\alpha}$, which by Lemma 3.1(4) is of order 7 and contained in $Q_{\beta}$, we have $\left|U_{\alpha}\right|=7$ and $U_{\alpha} \leq Q_{\beta}$. Suppose $U_{\alpha}=Z_{\beta}^{g}$ for some $g \in G$. Then $C_{G}\left(U_{\alpha}\right) \leq N_{\beta}^{g}$. But $Z_{\alpha} U_{\alpha} K_{\alpha} \leq C_{G}\left(U_{\alpha}\right)$ and $K_{\alpha}$ acts irreducibly on $Z_{\alpha}$. This shows that $Z_{\alpha} \leq Q_{\beta}^{g}$ and that $K_{\alpha} \cong K_{\alpha} Q_{\beta}^{g} / Q_{\beta}^{g} \cong S L_{2}(7)$ does not act irreducibly on $Q_{\beta}^{g} / Z_{\beta}^{g}=Q_{\beta}^{g} / U_{\alpha}$, a contradiction to Lemma 2.1(2).

For $\gamma \in\{\alpha, \beta\}$ let $X_{\gamma}=C_{N_{\gamma}}\left(U_{\alpha}\right)$ and $Y_{\gamma}=N_{N_{\gamma}}\left(U_{\alpha}\right)$. Let $X=$ $\left\langle X_{\alpha}, X_{\beta}\right\rangle, Y=\left\langle Y_{\alpha}, Y_{\beta}\right\rangle$, and let $X_{0}=C_{G}\left(U_{\alpha}\right), Y_{0}=N_{G}\left(U_{\alpha}\right)$. Obviously,

$$
X \leq X_{0} \cap Y \leq X_{0} Y \leq Y_{0} .
$$

Lemma 4.2. Let $C=C_{Q_{\beta}}\left(U_{\alpha}\right)$. Then $C \in \operatorname{Syl}_{7}(A)$ for $A \in\{X, Y$, $\left.X_{0}, Y_{0}\right\}$; in particular, the 7-part of the order of $A$ is $7^{4}$.

Proof. We first note that $|C|=7^{4}$ by Lemma 4.1 and as $Q_{\beta}$ is extraspecial. Also, since $C \leq X,(*)$ shows that it suffices to prove the statement for $A=Y_{0}$. Let $R \in \operatorname{Syl}_{7}\left(Y_{0}\right)$. We may assume that $C \leq R$, so $R \cap Q_{\beta}=C$. Since $U_{\alpha}$ is not 7-central by Lemma 4.1 , we have $|R| \leq 7^{5}$, so $|R: C| \leq 7$ and $C \unlhd R$. Hence also $Z_{\beta}=Q_{\beta}^{\prime}=C^{\prime} \unlhd R$ and therefore $R \leq N_{G}\left(Z_{\beta}\right)=N_{\beta}$. If $|R|=7^{5}$, then $R \not \leq Q_{\beta}$ and so $C \unlhd Q_{\beta} R=R_{1} \in \operatorname{Syl}_{7}\left(N_{\beta}\right)$. But then $U_{\alpha} Z_{\beta}=Z(C) \unlhd R_{1}$ and Lemma 2.1 applied to $Q_{\beta} / Z_{\beta}$ implies that $Z(C)^{g}=Z_{\alpha}$ for $g \in N_{\beta}$ with $R_{1}^{g}=S$. As all elements in $Z_{\alpha}^{\#}$ are 7 -central we get a contradiction to Lemma 4.1. So $R=C$ and we are done.

Lemma 4.3. (1) $X_{\alpha}=U_{\alpha} \times Z_{\alpha} K_{\alpha} \cong 7 \times 7^{2}: S L_{2}(7)$ and $Y_{\alpha}=$ $U_{\alpha} Z_{\alpha} J_{\alpha} \sim\left(7 \times 7^{2}: S L_{2}(7)\right): 6$. 
(2) $X_{\beta} \sim 7 \times 7_{+}^{1+2} \cdot(\operatorname{Sym}(3) \times 3)$ and $Y_{\beta} \sim\left(7 \times 7_{+}^{1+2}\right) .(\operatorname{Sym}(3) \times 3 \times 6)$; in particular, $O_{7^{\prime}}\left(X_{\beta}\right)=1$.

(3) $X_{\beta}$ acts irreducibly on $O_{7}\left(X_{\beta} / U_{\alpha} Z_{\beta}\right)$ and $\left|X_{\beta}: X_{\beta} \cap L_{\beta}\right|=6$.

Proof. (1) is a straightforward consequence of the definition of $U_{\alpha}$ and (2) follows easily from Lemmas 4.1, 4.2, and 2.1. (Note that $t_{1}, t_{3} \in\left(Y_{\alpha} \cap\right.$ $\left.Y_{\beta}\right) \backslash\left(X_{\alpha} \cup X_{\beta}\right)$.) Finally, the first statement of (3) holds by Lemma 2.1(6), the second one by (2) and Lemma 2.1(4).

LEMMA 4.4. $N_{X_{0}}(C)=X_{\beta}=N_{X}(C)$.

Proof. Let $N=N_{X_{0}}(C)$. As $C \leq Q_{\beta}$ and $|C|=7^{4}, C^{\prime}=Z_{\beta}$ and therefore

$$
N \leq N_{X_{0}}\left(C^{\prime}\right)=N_{X_{0}}\left(Z_{\beta}\right) \leq N_{G}\left(Z_{\beta}\right)=N_{\beta} .
$$

Since $U_{\alpha} \leq Z\left(X_{0}\right)$ we get $N \leq C_{N_{\beta}}\left(U_{\alpha}\right)=X_{\beta}$ and so $N_{X_{0}}(C)=X_{\beta}=$ $N_{X}(C)$.

Proposition 4.5. $C_{G}\left(U_{\alpha}\right)=X \cong 7 \times H e$ and $N_{G}\left(U_{\alpha}\right)=Y \sim(7:$ $3 \times H e): 2$; in particular, $N_{G}\left(U_{\alpha}\right)$ is not 7 -constrained and $G$ is not of local characteristic 7 .

Proof. With $C_{G}\left(U_{\alpha}\right)=X_{0}$ as above, by Lemma 4.2 we have $C=$ $C_{Q_{\beta}}\left(U_{\alpha}\right) \in S y l_{7}\left(X_{0}\right)$. We are going to apply Corollary 2.4 and Lemma 2.7.

Let $K>U_{\alpha}$ be a subgroup of $X_{0}$ which is normalized by $X$. Suppose first $K=U_{\alpha} \times O$, where $O=O_{7^{\prime}}(K)$. Then

$$
O=\left\langle C_{O}(z) \mid z \in Z_{\alpha}^{\#}\right\rangle
$$

Since all elements in $Z_{\alpha}$ are conjugate in $X$ into $Z_{\beta}$, we may assume that $C_{O}\left(Z_{\beta}\right) \neq 1$. But $C_{O}\left(Z_{\beta}\right) \unlhd X_{\beta}$ and so $C_{O}\left(Z_{\beta}\right) \leq O_{7^{\prime}}\left(X_{\beta}\right)=1$, a contradiction.

Hence $7^{2}$ divides $|K|$ and $K \cap C>U_{\alpha}$. Since $Z(C)$ is not normal in $X_{\alpha}$ we have $K \neq Z(C)$ and then Lemma 4.3(3) shows that $C \leq K$. Now $X_{\alpha}$ is generated by its Sylow 7-subgroups and so $X_{\alpha} \leq K$, too. Finally, $X_{\alpha} \cap X_{\beta} \sim$ $7 \times 7_{+}^{1+2} \cdot(2 \times 3)$ which shows that $X_{\beta}$ is generated by its conjugates of $X_{\alpha} \cap X_{\beta}$. We thus get $X=\left\langle X_{\alpha}, X_{\beta}\right\rangle \leq K$.

This shows that $F=X_{0} / U_{\alpha}$ and $H=X / U_{\alpha}$ satisfy the hypothesis of Lemma 2.7. So Lemma 2.7 tells us that $X_{0}$ has a unique normal subgroup $N$ which is minimal with respect to properly containing $U_{\alpha}$, that $N / U_{\alpha}$ is simple, and as $N / U_{\alpha}$ is obviously not cyclic, that $X_{0} / U_{\alpha}$ embeds into 
$\operatorname{Aut}\left(N / U_{\alpha}\right)$. Furthermore, as $N / U_{\alpha} \geq X / U_{\alpha}$, Lemma 4.4 and Corollary 2.4 imply that $N / U_{\alpha} \cong H e$. Since the Schur multiplier of $H e$ is trivial (see [5, page 104]) this implies $N \cong 7 \times H e$. Moreover, [5, page 104] reveals that $X_{\alpha}$ and $X_{\beta}$ are both maximal subgroups of $N$ and so $N=\left\langle X_{\alpha}, X_{\beta}\right\rangle=X$. Finally, $X_{0}=N N_{X_{0}}(C)=N N_{X}(C)=X$ by a Frattini argument and Lemma 4.4.

From the definition of $Y$ and the structures of $Y_{\alpha}, Y_{\beta}$ we further see that $X \unlhd Y$ and that $Y=\langle X, x\rangle$ for some element $x$ of order 6 whose cube does not centralize $X^{\prime}$. Together with the fact that $|\mathrm{Out}(\mathrm{He})|=2$ this shows that the structure of $Y$ is also as stated and that $Y=Y_{0}$. (Otherwise we would get $X_{0}>X$.)

Comparing Proposition 4.5 with the list of $p$-local subgroups of the monster $M$ in [5, page 234] we see that $Y$ is isomorphic to a $7 A$-normalizer in $M$.

\section{$\S 5$. 2-Central involutions}

The main goal of this section is to prove that $G$ contains a unique conjugacy class of 2-central involutions and to show that the centralizer of such an involution is of shape $2_{+}^{1+24} \cdot \mathrm{Co}_{1}$. As a consequence, we also get that the 2-part of $|G|$ is $2^{46}$.

Recall our fixed complement $T \cong 6 \times 6$ of $S$ in $N_{\alpha \beta}$ and its three involutions $t_{1}, t_{2}, t_{3}$.

Lemma 5.1. $t_{1}, t_{2}$, and $t_{3}$ are all conjugate in $G$.

Proof. By Lemma 3.2 and Corollary 2.2, $t_{2}$ and $t_{3}$ both project to a permutation conjugate to $(12)(34)(56)$ in $\left\langle L_{\beta}, t_{2}\right\rangle / O_{7,2}\left(\left\langle L_{\beta}, t_{2}\right\rangle\right)$. In particular, they are conjugate in $N_{\beta}$. Further, it follows from the structure of $G L_{2}(7)$ that there is some $g \in N_{N_{\alpha}}(T) \backslash N_{\alpha \beta}$ such that $Z_{\alpha}=Z_{\beta} Z_{\beta}^{g}$. As $\left[t_{1}, Z_{\beta}\right]=1 \neq\left[t_{1}, Z_{\alpha}\right]$ we get $\left[t_{1}, Z_{\beta}^{g}\right] \neq 1$ and so $t_{1} \neq t_{1}^{g}$. Since $g$ normalizes $\left\langle t_{1}, t_{2}\right\rangle=\left\langle t_{1}, t_{3}\right\rangle$ and $t_{2}^{g}=t_{2}$ we must have $t_{1}^{g}=t_{3}$ and the lemma follows.

Set $E=C_{Q_{\beta}}\left(t_{2}\right)$.

Lemma 5.2. $|E|=7^{2}$ and $C_{N_{\beta}}\left(t_{2}\right)$ has two orbits $\left\{U_{i} \mid i=0,1,2,3\right\}$ and $\left\{Z_{i} \mid i=0,1,2,3\right\}$ on the set of cyclic subgroups of order 7 in $E$, where $U_{0}=U_{\alpha}$ and the $Z_{i}$ are conjugates of $Z_{\beta}$. 
Proof. We have

$$
Q_{\beta} / Z_{\beta}=C_{Q_{\beta} / Z_{\beta}}\left(t_{2}\right) \oplus\left[Q_{\beta} / Z_{\beta}, t_{2}\right]
$$

as a decomposition invariant under $C_{N_{\beta}}\left(t_{2}\right)$. Since

$$
C_{\left\langle L_{\beta}, t_{2}\right\rangle}\left(t_{2}\right) Q_{\beta} / Q_{\beta} \sim 2 \times 2 \cdot \operatorname{Sym}(4)
$$

and $t_{1}$ inverts $Q_{\beta} / Z_{\beta}$ we conclude that both factors must be 2 -dimensional. So $|E|=7^{2}$ as $Z_{\beta} \not \leq E$.

Clearly $U_{0}=U_{\alpha} \leq E$. Now let $g \in N_{\alpha}$ with $t_{1}^{g}=t_{3}$ as in the proof of Lemma 5.1 and choose $h \in\left\langle L_{\beta}, t_{2}\right\rangle$ with $t_{3}^{h}=t_{2}$. Then $t_{1}^{g h}=t_{2}$ and so $t_{2}$ centralizes $Z_{0}=Z_{\beta}^{g h}$. Since

$$
Z_{0}=Z_{\beta}^{g h} \leq Z_{\alpha}^{g h}=Z_{\alpha}^{h} \leq Q_{\beta}^{h}=Q_{\beta}
$$

we see that $Z_{0} \leq E$. Finally, the length of the orbits follows from the facts that $Z_{0}$ and $U_{0}$ are not conjugate in $G$ by Proposition 4.5 , that $E$ contains precisely eight cyclic subgroups of order 7 , and that $t_{1} \in N^{\prime}$ for any subgroup $N \leq C_{N_{\beta}}\left(t_{2}\right)$ with $\left|C_{N_{\beta}}\left(t_{2}\right): N\right|<4$.

Set $N_{0}=N_{G}\left(Z_{0}\right), L_{0}=C_{G}\left(Z_{0}\right), Q_{0}=O_{7}\left(N_{0}\right), J_{0}=C_{N_{0}}\left(t_{2}\right)$, and $K_{0}=J_{0}^{\infty}$. Then $J_{0} \sim(7 \times 2 \cdot \operatorname{Alt}(7)): 6$ and $K_{0} \cong 2 \cdot \operatorname{Alt}(7)$.

Let $K=C_{G}\left(t_{2}\right)$.

LEMmA 5.3. We have $X \cap K=C_{X}\left(t_{2}\right) \sim 7 \times 2_{+}^{1+6} . L_{3}(2)$,

$$
Y \cap K=C_{Y}\left(t_{2}\right) \sim\left(7: 3 \times 2_{+}^{1+6} \cdot L_{3}(2)\right) .2,
$$

and $\mathrm{O}_{2}(X \cap K) /\left\langle t_{2}\right\rangle$ is irreducible as module for $(Y \cap K) / \mathrm{O}_{2}(Y \cap K)$.

Proof. We know that $X \cap K \geq K_{\alpha} \cong S L_{2}(7)$ and so [5, page 104] shows that $X \cap K$ is as stated (since the other involution centralizer $2^{2} . L_{3}(4) .2 \leq$ $H e$ does not contain $\left.S L_{2}(7)\right)$. The structure of $Y \cap K$ follows immediately.

LEMMA 5.4. $E \in \operatorname{Syl}_{7}(K)$.

Proof. We have $E \leq K$ by the definition of $E$ and $K$. Let $E \leq E_{1} \leq$ $E_{0} \in \operatorname{Syl}_{7}(K)$ such that $E \unlhd E_{1}$. Then $C_{E}\left(E_{1}\right) \neq 1$ and by Lemma 5.2 we may assume that either $Z_{0} \leq Z\left(E_{1}\right)$ or $U_{\alpha} \leq Z\left(E_{1}\right)$. In the second case we get $E=E_{1}=E_{0}$ from Lemma 5.3. In the first case, as $t_{2} Q_{0} \in Z\left(L_{0} / Q_{0}\right)$, $t_{2}$ inverts $Q_{0} / Z_{0}$ whence $E_{1} \cap Q_{0}=Z_{0}$ and $E_{1} Q_{0}=E Q_{0} \in \operatorname{Syl}_{7}\left(L_{0}\right)$. So $E=E_{1}=E_{0}$ as $\left|S: Q_{\beta}\right|=7$. 
Corollary 5.5. $O_{7}(K)=1$.

Proof. This can easily be deduced from Lemmas 5.2, 5.3, and the structure of $J_{0}$.

Proposition 5.6. $K \sim 2_{+}^{1+24} . \mathrm{Co}_{1}$.

Proof. Let $R=O_{7^{\prime}}(K)$ and $\bar{K}=K / R$. Let $\bar{N}$ be a minimal normal subgroup of $\bar{K}$ and $N$ its full preimage in $K$. Then 7 divides $|\bar{N}|$. Now Lemmas 5.2 and 5.4 immediately imply that $E \leq N$ and that $\bar{N}$ is simple. From the structures of $X \cap K$ and $K_{0}$ we infer further that

$$
O^{7}(\bar{K})=\bar{N} \geq\left\langle X \cap K, K_{0}\right\rangle R / R .
$$

In particular, $C_{\bar{N}}\left(\overline{Z_{i}}\right) \cong 7 \times A l t(7)$ and $C_{\bar{N}}\left(\overline{U_{i}}\right) \sim 7 \times 2^{6} \cdot L_{3}(2)$ or $C_{\bar{N}}\left(\overline{U_{i}}\right) \cong$ $7 \times L_{3}(2)$ for $i=0, \ldots, 3$. Applying Theorem 2.5 to $\bar{N}$ now shows that $\bar{N} \cong C o_{1}$ and also that $C_{\bar{N}}\left(U_{i}\right) \cong 7 \times L_{3}(2)$ must hold for $i=0, \ldots, 3$. In particular, $R \neq\left\langle t_{2}\right\rangle$.

On the other hand, as $R=\left\langle C_{R}(e) \mid e \in E^{\#}\right\rangle$ and we also see that $C_{R}\left(Z_{i}\right)=\left\langle t_{2}\right\rangle$ and $C_{R}\left(U_{i}\right) \cong 2_{+}^{1+6}$ for $i=0, \ldots, 3$ we conclude that $R$ is a 2 -group of order $|R|=2^{25}$. Also, $t_{2} \in R^{\prime}$. Since $R /\left\langle t_{2}\right\rangle$ admits $\bar{N}$ faithfully and the dimension of the smallest nontrivial representation of $C o_{1}$ over $G F(2)$ is 24 , we get that $R$ is extraspecial with $R^{\prime}=\left\langle t_{2}\right\rangle$ and $R / R^{\prime}$ is irreducible as $\bar{N}$-module. Moreover, by [13] the 24-dimensional representation of $C_{1}$ over $G F(2)$ is absolutely irreducible and uniquely determined. So our representation of $\bar{N}$ on $R /\left\langle t_{2}\right\rangle$ must be identical to the known one which embeds $C o_{1}$ into $O_{24}^{+}(2)$ and $R$ must be extraspecial of +-type. Finally, we get $K=N$ since the outer automorphism group of $\mathrm{Co}_{1}$ is trivial (see [5, page 180]).

Corollary 5.7. Let $P \in S y l_{2}(K)$. Then $Z(P)=\left\langle t_{2}\right\rangle$ and $P \in$ $S^{S y l} l_{2}(G)$; in particular, $\left\{t_{2}^{g} \mid g \in G\right\}$ is the unique conjugacy class of 2central involutions in $G$.

Proof. Since $K / O_{2}(K)$ acts faithfully on $O_{2}(K)$ and $O_{2}(K) \leq P$ we have $Z(P) \leq C_{K}\left(O_{2}(K)\right) \leq O_{2}(K)$. So $Z(P) \leq Z\left(O_{2}(K)\right)=\left\langle t_{2}\right\rangle$, which is the first assertion. Now let $P \unlhd P_{1} \leq P_{0} \in \operatorname{Syl}_{2}(G)$. Then $P_{1} \leq N_{G}(Z(P))=$ $N_{G}\left(\left\langle t_{2}\right\rangle\right)=C_{G}\left(t_{2}\right)=K$ and so $P=P_{1}=P_{0}$. 


\section{§6. The centralizer of a non 2-central involution and the proof of Theorem 1.1}

To establish the isomorphism $G \cong M$ we also need to find an involution with centralizer isomorphic to $2 \cdot B M$ in $G$. By [13], as a $K / O_{2}(K)$-module, $\mathrm{O}_{2}(K) /\left\langle t_{2}\right\rangle$ is isomorphic to the Leech lattice reduced $\bmod 2$. Let $s \in$ $\mathrm{O}_{2}(K)$ be an element such that $s\left\langle t_{2}\right\rangle /\left\langle t_{2}\right\rangle$ corresponds to a vector of type 2. Then from the Atlas [5, page 180], we have that $s$ has order $2, C_{K}(s) \sim$ $2^{1+1+22} . \mathrm{Co}_{2}$ and $C_{K}(s) /\langle s\rangle \sim 2_{+}^{1+22} . \mathrm{Co}_{2}$ is perfect.

LEMMA 6.1. $s$ and $t_{2}$ are not conjugate in $G$.

Proof. Suppose that $s$ is conjugate to $t_{2}$. Then $C_{K}(s)=K \cap C_{G}(s)$ and

$$
C_{K}(s) \mathrm{O}_{2}\left(C_{G}(s)\right) / \mathrm{O}_{2}\left(C_{G}(s)\right) \cong \mathrm{Co}_{2},
$$

as $\mathrm{Co}_{2}$ is a maximal subgroup in $\mathrm{Co}_{1}$. Thus $\mathrm{O}_{2}\left(C_{K}(s)\right) \leq \mathrm{O}_{2}(K) \cap$ $\mathrm{O}_{2}\left(C_{G}(s)\right)$. But

$$
\left[O_{2}(K) \cap O_{2}\left(C_{G}(s)\right), O_{2}(K) \cap O_{2}\left(C_{G}(s)\right)\right] \leq\left\langle t_{2}\right\rangle \cap\langle s\rangle=1
$$

so $\mathrm{O}_{2}(K) \cap \mathrm{O}_{2}\left(C_{G}(s)\right)$ is abelian. Since $\mathrm{O}_{2}\left(C_{K}(s)\right)$ is not abelian, we have a contradiction. Thus $s$ is not conjugate to $t_{2}$.

LEMmA 6.2. The elements of order 7 in $C_{G}(s)$ are not conjugate into $Z_{\beta}$. In particular, we can choose $s \in K=C_{G}\left(t_{2}\right)$ such that $s \in X$ and $C_{X}(s) \sim 7 \times 2^{2} \cdot L_{3}(4) .2$

Proof. Since all the involutions in $C_{G}\left(Z_{\beta}\right)=L_{\beta} \sim 7_{+}^{1+4} .2 \cdot A l t(7)$ are conjugate to $t_{2}$, the first claim follows at once from Lemma 6.1. Consequently, since 7 divides $\left|\mathrm{Co}_{2}\right|$, Lemmas 5.2 and 5.4 imply that we may choose $s \in O_{2}\left(C_{K}\left(t_{2}\right)\right)$ so that $\left[s, U_{\alpha}\right]=1$. Now $C_{X}(s) \sim 7 \times 2^{2} \cdot L_{3}(4) .2$ follows from [5, page 104] and Lemmas 5.3 and 6.1 .

Set $L=C_{G}(s)$, let $F_{0} \in S y l_{7}(X \cap L)$ and $F_{0} \leq F \in S y l_{7}(L)$. Observe that $\left|F_{0}\right|=7^{2}$.

Lemma 6.3. $F=F_{0}$ and all the elements of $F$ are conjugate in $L$ to elements of $U_{\alpha}$. 
Proof. We have already seen that $F$ does not contain any 7-central element. In particular, the same holds for $F_{0}$. Since by Lemma $4.2 C_{Q_{\beta}}\left(U_{\alpha}\right) \in$ $\operatorname{Syl}_{7}(X)$ and as by Lemma 3.1 all elements in $Q_{\beta}$ are either 7-central or conjugate into $U_{\alpha}$, we conclude that all elements in $F_{0}$ must be conjugate into $U_{\alpha}$. Thus, Sylow 7-subgroups of $C_{G}(f)$ are isomorphic to $C_{Q_{\beta}}\left(U_{\alpha}\right) \cong 7 \times 7_{+}^{1+2}$ for any $f \in F_{0}^{\#}$. We hence conclude that any 7 -group containing $F_{0}$ properly contains 7 -central elements and that $F=F_{0}$.

Finally, let $f \in F^{\#}$ and $g \in G$ with $f \in U_{\alpha}^{g}$. Then $s, s^{g^{-1}} \in C_{G}\left(U_{\alpha}\right)=$ $X$. Since by [5, page 104] He just contains two conjugacy classes of involutions and as $s$ is not conjugate to $t_{2}$ there must be some $h \in X$ such $s^{h}=s^{g^{-1}}$. Then $h g \in L$ and $U_{\alpha}^{h g}=U_{\alpha}^{g}=\langle f\rangle$.

Lemma 6.4. $C_{X}(s) \cap C_{K}(s)$ contains a Sylow 2-subgroup $V$ of $C_{X}(s)$.

Proof. From the identification of $\mathrm{O}_{2}(K)$ in Proposition 5.6, we have that $C_{O_{2}(K)}\left(U_{\alpha}\right) \sim 2_{+}^{1+6}$ and so $C_{O_{2}\left(C_{K}(s)\right)}\left(U_{\alpha}\right)$ has order $2^{6}$. Now, by [5, page 154], the centralizer of a Sylow 7-subgroup in $C_{K}(s) / O_{2}\left(C_{K}(s)\right) \cong \mathrm{Co}_{2}$ is $7 \times \operatorname{Dih}(8)$. Thus we infer that $C_{C_{K}(s)}\left(U_{\alpha}\right) \sim 7 \times V$ where $V$ has order $2^{9}$. By Lemma 6.2, $\left|C_{X}(s)\right|_{2}=2^{9}$. This proves that $V \in S y l_{2}\left(C_{X}(s)\right)$.

Proposition 6.5. $L \cong 2 \cdot B M$.

Proof. Clearly, $O_{7}(L)=1$. Let $U=O_{7^{\prime}}(L), \bar{L}=L / U$, let $\bar{N}$ be a minimal normal subgroup of $\bar{L}$, and $N$ its full preimage in $L$. Since 7 dives $|\bar{N}|, F \cap N \neq 1$ and so $F \leq N$, as all cyclic 7 -subgroups of $F$ are conjugate in $L$ by Lemma 6.3. A similar argument shows that $\bar{N}$ must be simple.

By Lemmas 6.2 and 6.3 we now have $C_{\bar{N}}(\bar{f}) \sim 7 \times 2 . L_{3}(4) .2$ or $7 \times$ $L_{3}(4) .2$ for any $f \in F^{\#}$.

In the first case, Theorem 2.6 gives $\bar{N} \cong B M$. Furthermore, $L \cap$ $C_{G}(F) \leq N$ and so $L / N$ embeds into $\operatorname{Out}(\bar{N})$. Since $\operatorname{Out}(B M)=1$ by [5, page 219] we get $L=N$. Also, as $U=\left\langle C_{U}(f) \mid f \in F^{\#}\right\rangle$ and now $C_{U}(f)=\langle s\rangle$ for any $f \in F^{\#}$ we get $U=\langle s\rangle$. Hence $L \cong 2 \cdot B M$ since $s \in C_{X}(s)^{\prime} \leq L^{\prime}$.

It remains to exclude the second case, that is, that $2^{2} \cong O_{2}(X \cap L)=U$. Now $U \leq V \leq C_{K}(s)$ where $V \in S y l_{2}\left(C_{X}(s)\right)$ as in Lemma 6.4. As $U$ is normalized by $C_{K}(s)$ we infer that $U=\left\langle s, t_{2}\right\rangle$. On the other hand, by $[5$, page 104] all involutions in $\mathrm{O}_{2}(X \cap L)$ are conjugate in $X$. This contradiction to Lemma 6.1 completes the proof.

Proof of Theorem 1.1. This now follows from Propositions 5.6, 6.5, [12], and $[25]$. 
We remark that in [25] Wilson proved that $M$ contains just one conjugacy class of maximal 7-local subgroups which is not mentioned in [5]. This additional 7-local subgroup was discovered by Chat Yin Ho [14] it is isomorphic to $7^{2}: S L_{2}(7)$ and so does not contain a Sylow 7-subgroup of $M$.

Acknowledgement. We would like to thank the anonymous referee for his remarks which improved the proof of Theorem 2.3 and considerably simplified Section 5 .

\section{REFERENCES}

[1] J. L. Alperin, Local representation theory, volume 11 of Cambridge Studies in Advanced Mathematics, Cambridge University Press, Cambridge, 1986. Modular representations as an introduction to the local representation theory of finite groups.

[2] H. Bender, Finite groups with dihedral Sylow 2-subgroups, J. Alg., 70 (1981), no. 1, $216-228$.

[3] H. Bender and G. Glauberman, Characters of finite groups with dihedral Sylow 2-subgroups, J. Alg., 70 (1981), no. 1, 200-215.

[4] R. Brauer and M. Suzuki, On finite groups of even order whose 2-Sylow group is a quaternion group, Proc. Nat. Acad. Sci. U.S.A., 45 (1959), 1757-1759.

[5] J. H. Conway, R. T. Curtis, S. P. Norton, R. A. Parker, and R. A. Wilson, Atlas of finite groups, Clarendon Press, Oxford, 1985.

[6] D. Gorenstein, Finite Groups, Harper and Row, New York, 1968.

[7] D. Gorenstein and R. Lyons, The local structure of finite groups of characteristic 2 type, Mem. Am. Math. Soc. 42(276), 1983, vii+731.

[8] D. Gorenstein, R. Lyons, and R. Solomon, The Classification of the Finite Simple Groups, Number 2, volume 40 of Mathematical Surveys and Monographs, American Mathematical Society, Providence, RI, 1996.

[9] D. Gorenstein, R. Lyons, and R. Solomon, The Classification of the Finite Simple Groups, Number 3, volume 40 of Mathematical Surveys and Monographs, American Mathematical Society, Providence, RI, 1998.

[10] D. Gorenstein and J. H. Walter, The characterization of finite groups with dihedral Sylow 2-subgroups. I, J. Alg., 2 (1965), 85-151.

[11] D. Gorenstein and J. H. Walter, The characterization of finite groups with dihedral Sylow 2-subgroups. II, J. Alg., 2 (1965), 218-270.

[12] R. Griess, U. Meierfrankenfeld, and Y. Segev, A uniqueness proof for the Monster, Ann. Math., 130 (1989), 567-602.

[13] R. L. Griess, A remark about representations of .1, Comm. Algebra, 13 (1985), 835-844.

[14] C. Y. Ho, A new 7-local subgroup of the Monster, J. Algebra, 115 (1988), no. 2, $513-520$. 
[15] A. A. Ivanov, A geometric characterization of the Monster, Groups, combinatorics \& geometry (Durham, 1990), volume 165 of London Math. Soc. Lecture Note Ser., Cambridge Univ. Press, Cambridge (1992), pp. 46-62.

[16] A. A. Ivanov and U. Meierfrankenfeld, Simple connectedness of the 3-local geometry of the Monster, J. Algebra, 194 (1997), no. 2, 383-407.

[17] C. Jansen, K. Lux, R. Parker, and R. Wilson, An atlas of Brauer characters, volume 11 of London Mathematical Society Monographs, New Series, The Clarendon Press Oxford University Press, New York, 1995. Appendix 2 by T. Breuer and S. Norton, Oxford Science Publications.

[18] P. B. Kleidman, The maximal subgroups of the Chevalley groups $G_{2}(q)$ with $q$ odd, the Ree groups ${ }^{2} G_{2}(q)$, and their automorphism groups, J. Algebra, 117 (1988), no. $1,30-71$.

[19] H. Kurzweil and B. Stellmacher, Theorie der endlichen Gruppen, Springer-Verlag, Berlin, 1998. Eine Einführung. [An introduction].

[20] U. Meierfrankenfeld, B. Stellmacher, and G. Stroth, Finite groups of local characteristic p: An overview, Groups, combinatorics \& geometry (Durham, 2001), World Sci. Publishing, River Edge, NJ (2003), pp. 155-192.

[21] C. W. Parker and P. J. Rowley, A characteristic 5 identification of the Lyons group, J. London Math. Soc. (2), 69 (2004), no. 1, 128-140.

[22] C. W. Parker and P. J. Rowley, Symplectic Amalgams, Springer, London, 2002.

[23] C. W. Parker and C. B. Wiedorn, A 5-local identification of the Monster, Arch. Math., 83 (2004), 404-415.

[24] C. W. Parker and C. B. Wiedorn, 5-local identifications of the Harada Norton group and of the Baby Monster, in preparation (2003).

[25] R. A. Wilson, The odd local subgroups of the Monster, J. Austral. Math. Soc. Ser. A, 44 (1988), no. 1, 1-16.

C. W. Parker

School of Mathematics and Statistics

University of Birmingham

Edgbaston

Birmingham B15 2TT

$U K$

C.W.Parker@bham.ac.uk

C. B. Wiedorn

School of Mathematics and Statistics

University of Birmingham

Edgbaston

Birmingham B15 2TT

$U K$

wiedornc@for.mat. bham.ac.uk 